\title{
Teaching psychiatry to undergraduate medical students in Somalia
}

\author{
Lauren Gavaghan, ${ }^{1}$ Peter Hughes, ${ }^{2}$ Khalid Saeed ${ }^{3}$ and Susannah Whitwell ${ }^{4}$
}

'Specialist Registrar in Psychiatry, Co-Lead of the King's-THET Somaliland Partnership Mental Health Group, South London and Maudsley Trust, London, UK, UK, email laureninsomaliland@ gmail.com

${ }^{2}$ Consultant Psychiatrist, Springfield University Hospital, London, UK

\section{London, UK}

${ }^{3}$ Regional Advisor, Mental Health and Substance Abuse World Health Organization Regional Office for the Eastern Mediterranean, Cairo, Egypt ${ }^{4}$ Consultant Psychiatrist, South London and Maudsley NHS Foundation Trust, Clinical Lead, King's THET Somaliland Partnership, King's Centre for Global Health, King's College London, UK
This paper describes a pilot project in which (for the first time, worldwide) psychiatry was taught to undergraduate medical students in Somalia using an evidence-based intervention - the World Health Organization's Mental Health Gap Action Programme Intervention Guide.

In the Eastern Mediterranean Region (EMRO) of the World Health Organization (WHO), which spans from Morocco to Pakistan and which includes Somalia, $2.8 \%$ of training for medical doctors and $3.4 \%$ of training for nurses is devoted to psychiatry and other subjects related to mental health. These are rates similar to those for the rest of the world. Across the region, as in the rest of the world, 6.11 nurses and 3.28 doctors per 100000 population per year are trained in mental health. Compared with nurses and non-psychiatric doctors, few psychiatrists, psychologists and social workers specialise each year (WHO, 2011). It is therefore unlikely that mental healthcare will be accessible or available to the vast majority of the populations in need if the current reliance on in-service training is continued. Somaliland is an impoverished area. The health service, including mental health services, has low capacity. There are three in-patient units in the country, minimal community services and limited psychiatric medications, with services located only in the main towns (WHO, 2006).

The WHO launched the Mental Health Gap Action Programme (mhGAP) with the aim of closing the gap between need and services available for mental, neurological and substance use disorders in low- and middle-income countries. Although these disorders account for $14 \%$ of the global burden of disease, up to $75 \%$ of people in low-income countries do not have access to appropriate treatment (WHO, 2008).

The Mental Health Gap Intervention Guide (mhGAP-IG) was developed as a technical tool. It is an evidence-based, peer-reviewed manual to guide health practitioners through the assessment and management of mental health conditions at a primary-care level (Barbui et al, 2010; WHO, 2010).

Training non-specialists in mental healthcare is vital, due to the lack of mental health professionals in a region like Somaliland, but also due to wider issues. The WHO emphasises the integration of mental health into primary care as the most viable way to close the existing treatment gap in mental health. Wider advantages of this approach include better holistic care, wider accessibility of services, less stigma and therefore better acceptability, and ultimately the production of better health outcomes at lower cost (WHO \& World Organization of Family Doctors, 2008).

This report describes the experience gained through the King's-THET Somaliland Partnership (KTSP) (Leather et al, 2006) of incorporating the mhGAP-IG into undergraduate medical student teaching at Amoud University, Borama and Hargeisa University, Hargeisa, in the Somaliland region of Somalia in May 2012 and May 2013. This was the first time worldwide that the mhGAP-IG has been utilised in this way in medical undergraduate teaching. The objectives of this pilot were to incorporate the mhGAP-IG into Somaliland medical student undergraduate psychiatry training as an additional resource to aid understanding of the assessment and management of mental disorders and to further develop and evaluate this teaching using the mhGAP-IG.

\section{Methods}

The basic psychiatry teaching course offered to medical students by KTSP in collaboration with local partners is an annual intensive 9-day programme. In 2012, it ran 15-24 May and was held at Amoud University, Borama. In 2013, it ran 6-15 May and was held at Hargeisa Group Hospital, Hargeisa. Students in their penultimate year of medical school attended from both the Universities of Amoud and Hargeisa. Thirty-six medical students and one nursing student took part in 2012, and 52 medical students in 2013. The mhGAP-IG was incorporated into the curriculum primarily to explore the management of conditions and to familiarise students with the manual.

The teaching was delivered using different techniques, including lectures and more interactive methods already piloted in this environment with good effect on knowledge and attitudes (Syed Sheriff et al, 2010). The mhGAP-IG was used in its current format. Each student received a copy of the manual and the teaching was added to the existing lecture-based teaching. Role-play and case-based discussions were used, with the mhGAP-IG as the basis to guide learning. Aetiology of disorders was covered separately from the mhGAP-IG, as it is primarily a tool for assessment, diagnosis and management.

There was a need to adapt teaching using the mhGAP-IG to ensure relevance to the Somaliland context. For example, because of the widespread use of khat in Somaliland and its effects on mental health, discussion of this issue was warranted. Not all medications are available in the country, such 


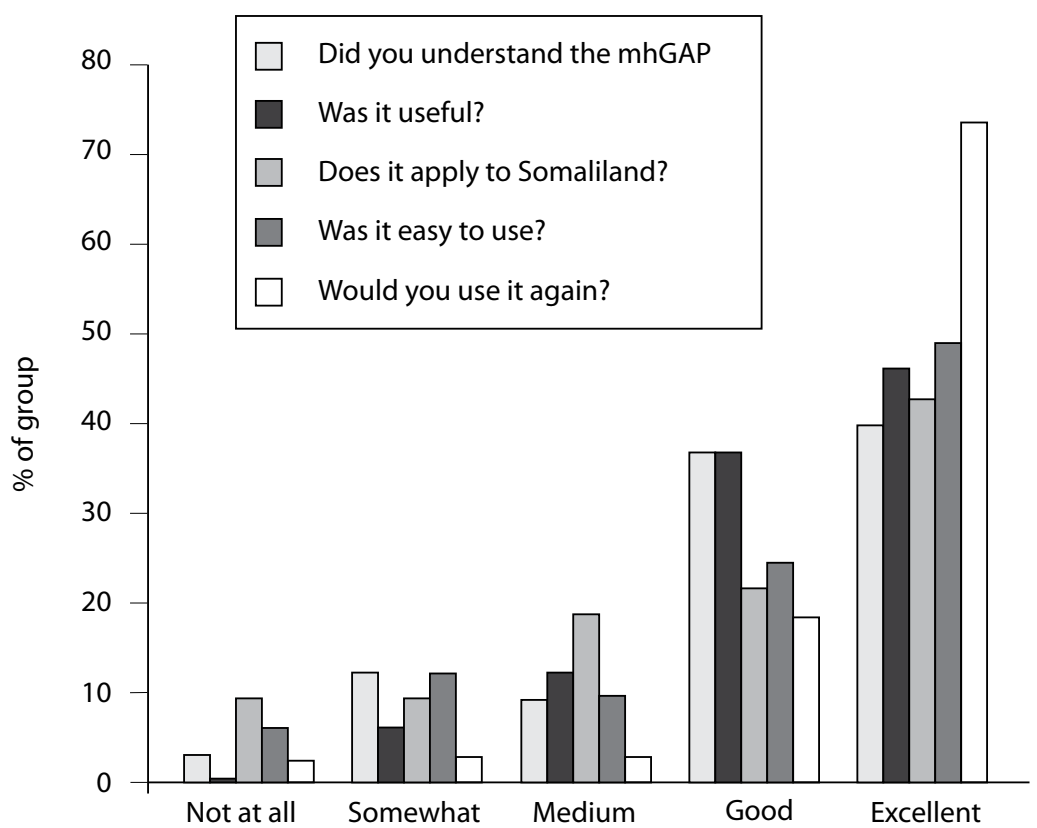

Fig. 1

Survey of the use of the mhGAP-IG in medical student undergraduate psychiatry teaching in Somaliland (2012) as lithium and medications for behavioural disorders, and this required consideration.

Following the course, a short satisfaction survey was conducted to evaluate participants' views of the mhGAP and its inclusion in teaching. Participants were asked to answer five questions relating to their experience of the mhGAP-IG training:

- Did you understand the mhGAP?

- Was it useful?

- Does it apply to Somaliland?

- Was it easy to use?

- Would you use it again?

Answers to each of the five questions were ranked on a five-point Likert scale, ranging from 'not at all' to 'excellent'.

Improvements in mhGAP-based knowledge were quantified in 2013, although they were not tested during the initial pilot in 2012. A draft of the WHO mhGAP Monitoring and Evaluation Toolkit's Pre- and Post-Test for the mhGAP Base Course (WHO, 2013) was piloted in May 2013, to test students' knowledge immediately before and after the 2 weeks of training. This included 25 multiple-choice questions directly related to $\mathrm{mh}$ GAP-IG information. The KTSP course evaluation also includes pre- and post-course questionnaires of attitudes towards mental illness and these, along with the mhGAP knowledge test, have informed further development of the mhGAP training.

\section{Results}

Thirty-three of the 36 medical students responded to the survey in 2012 (Fig. 1). Fifty-one out of the 52 students responded in 2013 (Fig. 2). Ability to understand the mhGAP-IG in 2012 was rated as mostly 'good' or 'excellent' (36\% and 39\% respectively). In 2013, this proportion had risen substantially, with $75 \%$ of students ranking their understanding as 'excellent' and $24 \%$ as 'good'. In terms of its usefulness, in 2012, $82 \%$ of students felt it to be good or excellent, compared with $94 \%$ of students in 2013. Importantly, in relation to its relevance to Somaliland from the viewpoint of the medical students, in $201242 \%$ rated it 'excellent' and approximately $40 \%$ rated it to be either 'good' or 'medium'. In $2013,49 \%$ of students ranked its appropriateness to Somaliland 'excellent' and $24 \%$ 'good'. Nearly half of the students rated the mhGAP-IG 'excellent' in terms of its ease of use in 2012, with this proportion increasing to $69 \%$ in 2013 , and nearly a quarter rated it 'good' in 2012 (18\% in 2013). The majority of students were keen to use mhGAP-IG again, with $73 \%$ rating this as 'excellent' in 2012 (78\% in 2013) and $18 \%$ rating it as 'good' (16\% in 2013).

To demonstrate changes in knowledge, the mhGAP pre- and post-test was completed by the medical students immediately before the course (with most of them having had an online copy of the mhGAP-IG in advance) and at the end of the 2-week course. Of the 52 students, 48 completed the post-course test. 
The test results showed objective improvements in knowledge, with the range of results increasing from $28-84 \%$ pre-course to $68-100 \%$ post-course. Only two students scored less in their post-test than in their pre-test (Table 1).

\section{Discussion and conclusions}

The incorporation of the mhGAP-IG into medical undergraduate teaching in Somaliland was received well during training in 2012 and 2013. There was increased understanding of the mhGAP-IG after the second period of training, both as subjectively perceived by medical students and as objectively shown by improvements on the postcourse mhGAP-IG knowledge tests. This may have been due to improved methods of delivering the training the second time, with more interactive methods used to demonstrate use of the manual and each student having access to an individual copy of the mhGAP-IG.

The risk of social desirability bias may be a limitation of this type of study, although the fact that a few students reported that they did not find the mhGAP wholly useful suggests this bias was not substantial.

It is neither feasible, nor sustainable, to rely wholly on specialists to deliver mental healthcare to patients and this is highly relevant in the Somaliland region of Somalia, where mental health services are very limited.

We feel it is important and timely to roll out the inclusion of the mhGAP-IG into both the medical and nursing undergraduate curriculums, so that those specialising in other fields may have at least an evidence-based grounding in the management of mental and substance use disorders.
The aim will be to continue this training and to supervise those in the country who are able to lead the teaching, in order to ensure sustainability. This would include monitoring of the incorporation of the mhGAP-IG and measuring future changes in skills rather than simply knowledge, which alone is insufficient to improve mental healthcare at a wider level.

\section{References}

Barbui, C., Dua, T., van Ommeren, M., et al (2010) Challenges in developing evidence-based recommendations using the GRADE approach: the case of mental, neurological, and substance use disorders. PLoS Medicine, 7(8), e1000322. doi:10.1371/journal. pmed.1000322.

Leather, A., Ismail, E. A., Ali, R., et al (2006) Working together to rebuild health care in post-conflict Somaliland. Lancet, 368 1119-1125.

Syed Sheriff, R., Baraco, A., Nour, A., et al (2010) Improving human resource provision for mental health in Somaliland. Psychiatric Services, 61, 225-227.

WHO (2006) AIMS Report on Mental Health System in Somaliland Region of Somalia: A Report of the Assessment of the Mental Health System in Somalia Using the World Health Organization Assessment Instrument for Mental Health Systems (WHO-AIMS). WHO Country Office Somalia, WHO Regional Office for the Eastern Mediterranean, WHO Department of Mental Health and Substance Abuse, Ministry of Health Republic of Somalia, Ministry of Health Somaliland.

WHO (2008) Mental Health Gap Action Programme (mhGAP). World Health Organization. At http://www.who.int/mental_health/ mhgap/

WHO (2010) mhGAP Intervention Guide for Mental, Neurological and Substance Use Disorders in Non-specialised Health Settings. World Health Organization.

WHO (2011) Mental Health Atlas: Eastern Mediterranean Regional Review. World Health Organization.

WHO (2013) Draft mhGAP Monitoring and Evaluation Toolkit: Preand Post-Test for mhGAP Base Course. World Health Organization.

WHO \& World Organization of Family Doctors (2008) Integrating Mental Health into Primary Care A Global Perspective. World Health Organization \& World Organization of Family Doctors (WONCA).

\section{Where There Is No Child Psychiatrist}

\section{A mental healthcare manual}

By Valsamma Eapen, Philip Graham and Shoba Srinath

Mental health problems and worries are common among infants, children and adolescents in every part of the world. This book is a practical manual for primary healthcare professionals, teachers and anyone who works with children - especially in places where specialist psychiatric care is not available.

After giving the reader an overview of child mental health problems, the manual goes on to deal with the various developmental, behavioural and emotional problems that arise in as many as $10 \%$ of the youth population. For each problem it first provides a case study and then

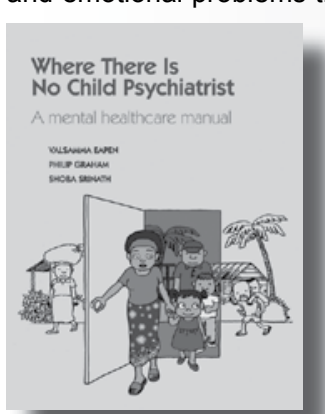
describes how to find out more about a child with this problem. It suggests what can be done to help the child and their family.

It also examines the mental health aspects of situations such as when children are maltreated or exposed to natural or man-made disasters.

- Covers problems that health workers and teachers in low- and middle-income countries often have to deal with.

- Written in simple language with many case examples and pictures.

- The only comprehensive book on mental healthcare in young people for those with no access to specialist medical advice.

Published Oct 2012, paperback, 214 pages, ISBN 978-1-908020-48-2, price £10 (RCPsych members' price: £8) 